\title{
Percutaneous transarterial balloon dilatation of the mitral valve: five year experience
}

\author{
Uros U Babic, Sreten Grujicic, Zoran Popovic, Zoran Djurisic, Petar Pejcic, \\ Mihailo Vucinic
}

\begin{abstract}
Objective-To examine the value of transarterial balloon dilatation of the mitral valve for treatment of patients with mitral stenosis over a period of five years.

Design-Analysis of patients' functional state, and haemodynamic and echocardiographic variables, before and immediately after the procedure and during a follow up of up to five years.

Setting-A cardiovascular centre in Belgrade, Yugoslavia.

Patients-Two hundred and ninety four patients who underwent percutaneous transarterial dilatation of the mitral valve between February 1985 and February 1990.

Results-Mean mitral valve area was enlarged by $109 \%$. Complications included death $(0.7 \%)$, severe mitral insufficiency $(2 \cdot 3 \%)$, mild mitral insufficiency $(9.9 \%)$, cerebral embolism $(2 \%)$, and injury to the femoral artery (2\%). Two more patients died at two and 11 months after the procedure. Late cardiac surgery was needed in eight patients (mitral insufficiency in three, restenosis in three, thrombus in one, and endocarditis in one. Restenosis occurred in seven patients. Four of these underwent repeat dilatation and three had surgery. Improvement of symptoms was seen in $94 \%$ of patients during the follow up.

Conclusion-Transarterial balloon dilatation of the mitral valve gave good results with acceptable morbidity and mortality and had some advantages over the anterograde approach.
\end{abstract}

Balloon dilatation of the mitral valve is often used to treat mitral valve stenosis. The original transvenous technique reported by Inoue et $a l^{1}$ has been modified by many workers. ${ }^{23}$ To avoid injuring the interatrial septum with large balloon catheters and to enable better stabilisation of the balloon across the mitral valve we started to use the transarterial approach in February 1985. This study describes our five year experience with this technique in 294 patients.

\section{Patients and methods}

PATIENTS

From February 1985 to February 1990, 294 patients $(238(81 \%)$ women and $56(19 \%)$ men aged 15-76 (mean (SD) 36 (7) years) underwent transarterial balloon dilatation of the mitral valve. Atrial fibrillation was present in $64 \%$ of patients. Only 35 patients $(12 \%)$ had been given anticoagulants before the procedure. Sixty one patients were in the New York Heart Association (NYHA) functional class IV, 185 in class III, 37 in class II, and 11 in class I (all young women who wished to become pregnant). According to the echocardiographic criteria ${ }^{4} 171(58 \%)$ patients had a pliable mitral valve. There was calcification of the mitral valve or mitral apparatus or both, as verified by echocardiography and fluoroscopy, in 49 patients $(16 \%)$ although 15 still had a pliable valve.

None of the patients had important coronary artery disease, 48 had aortic valve regurgitation of less than Sellers ${ }^{5}$ grade III, two had considerable and four minor aortic valve stenosis. Nine patients had undergone previous open or closed mitral commissurotomy.

Patients with left atrial thrombi or mitral valve regurgitation greater than grade II and those with a significant iliofemoral arterial disease were excluded. All patients gave informed consent.

\section{TECHNIQUE}

The technique of transarterial balloon dilatation of the mitral valve has been reported previously. ${ }^{67} \mathrm{~A}$ description of our present technique is given here. The double balloon catheter technique has not been used since low profile single catheter with the shaft connected to two balloons in parallel (twin Mansfield) became available.

Right and left catheterisations were performed. Cardiac output was determined by the Fick principle based on an assumed oxygen consumption ${ }^{8}$ and by the thermodilution technique. The mitral valve area was calculated from the Gorlin formula. ${ }^{9}$ Coronary angiography was performed in all patients to exclude those with coronary artery disease and to look for possible neovascularisation of left atrial thrombi. ${ }^{10} \mathrm{~A}$ Mullins 8 French trans-septal sheath was advanced from the right femoral vein trans-septally into the left atrium. Heparin (100 units $/ \mathrm{kg}$ ) was given. A 7 French retrieval catheter ${ }^{7}$ was advanced through a sheath from the left femoral artery to the aortic root where its wire loop was opened. A 7 French floating Swan-Ganz catheter (Vygon), which permits a 0.035 inch wire to pass through its central lumen, was advanced through the Mullins sheath into the left atrium. The Swan-Ganz floating catheter

11000 , Yugoslavia.

Accepted for publication 1 August 1991 
was inflated with carbon dioxide and advanced through the left ventricle into the aortic root across the wire loop of the retrieval catheter. A guide wire $(350 \mathrm{~cm}$ long 0.035 inch diameter, with flexible J end, Cook) was advanced via the Swan-Ganz floating catheter from the right femoral vein into the aortic root where it was snared by the retrieval catheter and brought out of the body through the left femoral artery. A F14 sheath was inserted over this wire into the left femoral artery. A balloon catheter (twin Mansfield) was introduced over the wire through the F14 sheath from the left femoral artery to the aortic root. The floating catheter was withdrawn and a two way Tuohy-Borst adapter was attached on the proximal hub of the Mullins sheath. The central channel of the adapter secured the venous end of the long guide wire while the sidearm served as the left atrial line for monitoring of left atrial pressure and for simultaneous injection of contrast medium into the left atrium. The long guide wire was pushed from both sides to form a large loop within the left ventricle. The balloon catheter was advanced over the loop into the left ventricle up to $2-3 \mathrm{~cm}$ from the apex after which it was placed across the mitral valve by pulling the long guide wire from the venous side while the arterial end of the long wire was secured to the balloon catheter shaft by a $\mathrm{Y}$ connector (fig 1).

After the balloon was positioned across the valve orifice it was inflated and optimal positioning of the balloon was controlled by injection of contrast medium into the left atrium ( $30 \mathrm{ml}$ volume; $8 \mathrm{ml} / \mathrm{s}$ flow). To keep the balloon stable, the Mullins sheath was

Figure 1 Diagram of transarterial balloon dilatation of the mitral valve. Note the tip of the Mullins sheath put into the left atrium during balloon inflation. APL, arterial pressure line from the sidearm of the introducer sheath; $B C$, balloon catheter; $L A L$, left atrial line; $L G W$, long guide wire; $M S H$, Mullins wire; MSH, Mullins
sheath; TBA, TuohyBorst two-way adapter.

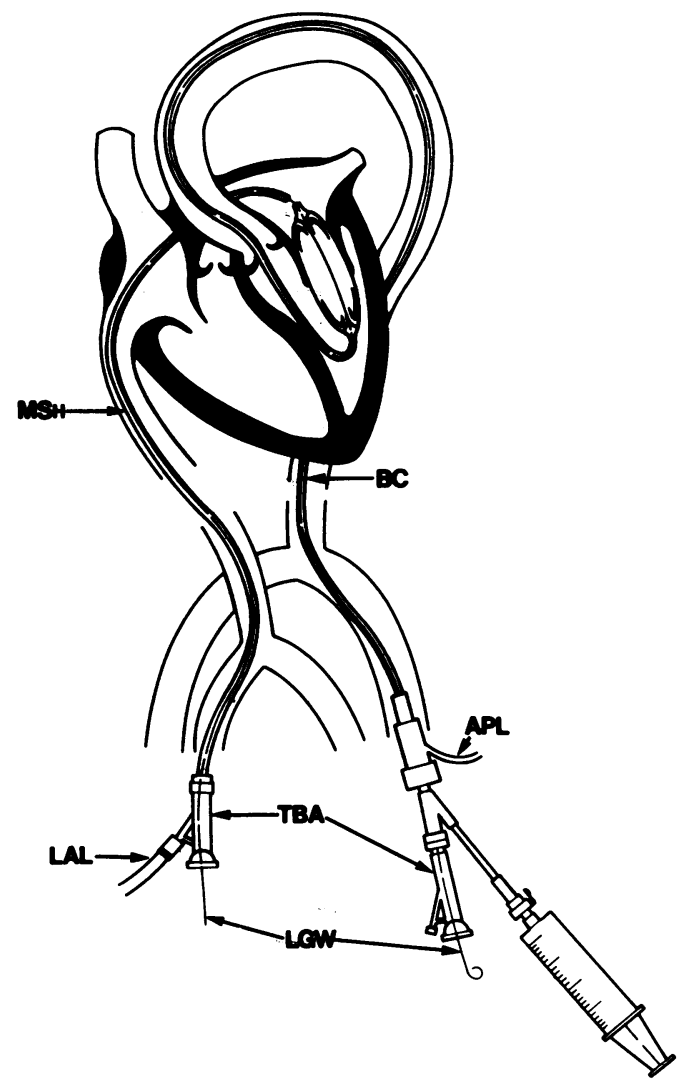

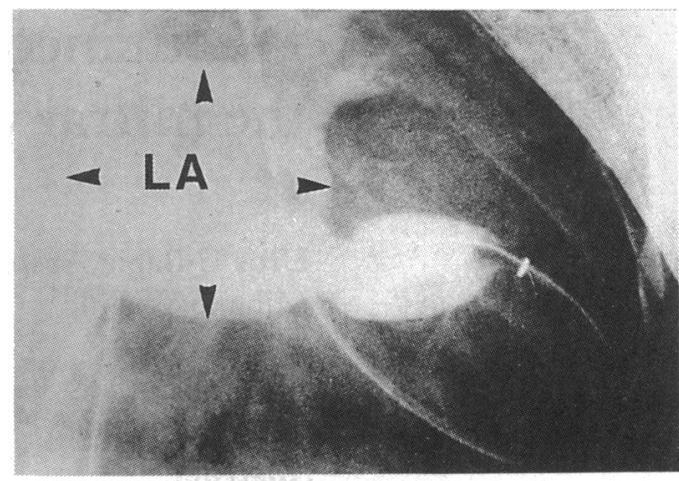

Figure 2 Cineangiogram showing a twin balloon $(18+20 \mathrm{~mm}$ Mansfield) placed and semi-inflated across the mitral valve.

pushed while the balloon catheter (secured to the wire by $\mathrm{Y}$ connector) was pulled forward (fig 2). The balloon was deflated and pulled back into the aortic root.

A single $25 \mathrm{~mm}$ balloon (Mansfield) was used in 17 patients, two balloons $(2 \times 18$, $18+20,2 \times 20 \mathrm{~mm}$ Mansfield) in 188, and a bifoil $(2 \times 19$ Schneider $)$ or a "twin" $(18+20)$ in 89 patients.

Left atrial pressure and the mitral gradients were recorded by advancing the Mullins sheath into the left ventricle and withdrawing it into the left atrium. When necessary, dilatation was repeated with the same or a larger balloon exchanged over the wire. The haemodynamic measurements and left ventriculography were repeated after final dilatation and in most patients contrast medium was injected into the left atrium through a pigtail catheter in the Mullins sheath to exclude left-right shunting. Cephalosporin was given intravenously one hour before and for the first 24 hours after the procedure. An oral antibiotic was given for the next five days. Oral anticoagulants were given to all patients for six months after balloon dilatation and treatment was continued for longer in those with atrial fibrillation. Balloon sizes were chosen according to diastolic left ventricular diameter, valve anatomy, and the body surface area.

\section{FOLLOW UP}

The patients were followed up as outpatients one and six months after the procedure and thereafter once a year. Clinical state and chest $x$ ray film were assessed and physical examination and cross sectional and Doppler echocardiography were performed at each visit. Repeat catheterisation was performed only in patients with significant recurrent symptoms.

DATA ANALYSIS

Data were expressed as a mean (SD). The variables were compared by Student's $t$ test.

\section{Results}

Within the five year period 16 patients with mitral stenosis were found to have left atrial thrombi and were sent for surgery rather than balloon dilatation of the valve. Transthoracic echocardiography showed atrial thrombi in 11 
Haemodynamic results before and after percutaneous transarterial balloon dilatation of the mitral valve in 294 patients

\begin{tabular}{llll}
\hline & $\begin{array}{l}\text { Before } \\
\text { mean }(S D)\end{array}$ & $\begin{array}{l}\text { After } \\
\text { mean }(S D)\end{array}$ & p Values \\
\hline Pressures (mm Hg): & & & \\
$\quad$ Pulmonary artery & $62(12)$ & $44(8)$ & $<0.001$ \\
$\quad$ Systolic & $23(6)$ & $11(4)$ & $<0.001$ \\
$\quad$ Mean left atrial & $18(6)$ & $6(3)$ & $<0.001$ \\
$\quad$ Mean mitral valve gradient & $4 \cdot 1(0.7)$ & $4.3(0.6)$ & $>0.05$ \\
Cardiac output $\left(1 /\right.$ min $^{2}$ & $1.1(0.3)$ & $2.3(0.4)$ & $<0.001$ \\
Mitral valve area $\left(\mathrm{cm}^{2}\right)$ & & & \\
\hline
\end{tabular}

patients, and coronary angiography detected neovascularisation of thrombi in eight, including five in whom echocardiography had been negative. Thrombi were found at operation in all of these patients. In four patients transseptal catheterisation caused a haemopericardium and cardiac tamponade that required subxiphoid pericardiocentesis with a pigtail catheter. These patients were discharged and the procedure was successfully repeated two to four months later. Valve dilatation was accomplished in all 294 patients in whom it was attempted. The time needed for the entire procedure with a single balloon catheter (bifoil or twin) was less than one hour in most patients. In 14 patients the result was poor (enlargement of the mitral valve area was less than $25 \%$ ).

The table gives the haemodynamic indices before and after balloon dilatation. The mean mitral valve area was enlarged by $109 \%$. The increase in valve area was more prominent in younger patients and in those with a pliable valve (fig 3 ). In two patients with concomitant aortic valve stenosis, combined aortic and mitral valve balloon dilatation was performed with the same long guide wire arrangement. ${ }^{11}$ The aortic valve gradients were reduced from 85 to $25 \mathrm{~mm} \mathrm{Hg}$ and 110 to $50 \mathrm{~mm} \mathrm{Hg}$ in these two patients. Aortic valve insufficiency grade II was produced in both.

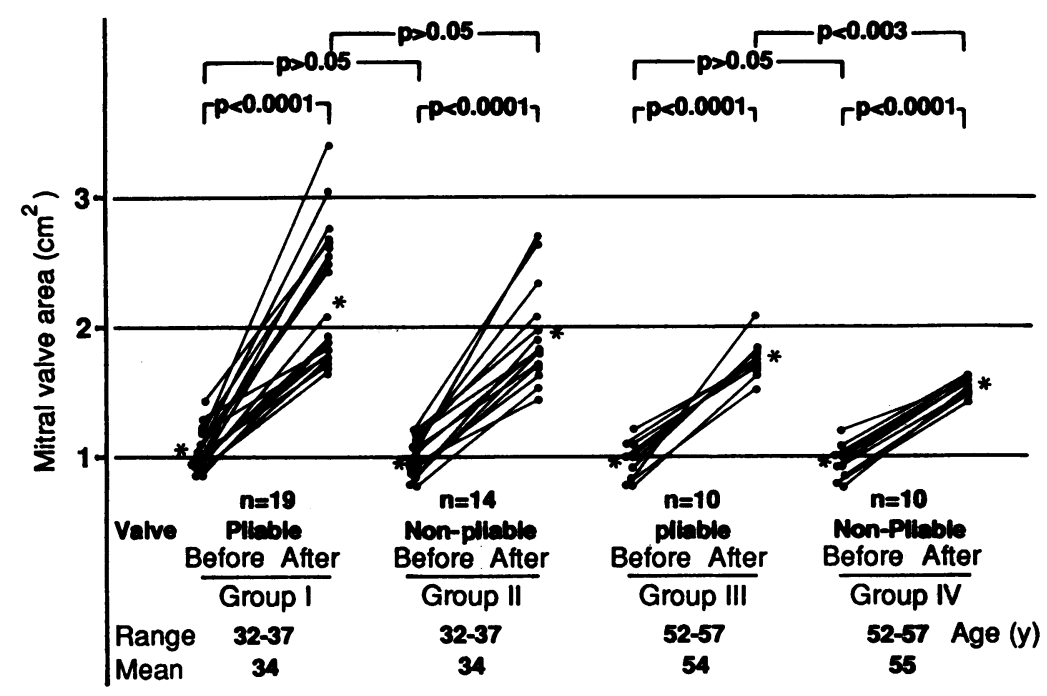

Figure 3 Individual values for mitral valve area before and after dilatation in 53 patients. All patients were dilated with the same $18+20 \mathrm{~mm}$ twin Mansfield balloon. Two age groups (range five years) were compared. The achieved enlargement of the mitral valve area was higher in younger patients and in those with a pliable valve.
COMPLICATIONS

There were two procedural deaths. One patient died of cerebral embolism and one of left coronary artery embolism. Cerebral embolism occurred in five further patients, only one of whom recovered completely. In all seven patients with embolic complications the transthoracic echocardiography had given inconclusive findings due to inadequate echogenicity, and transoesophageal echocardiography was not available at that time. Immediate surgery was needed in three patients because of acute severe mitral insufficiency, and at operation a tear in the anterior mitral leaflet was found in all of them. Four patients with post procedural grade III mitral insufficiency were discharged in a stable clinical condition. The left ventricular apex was perforated in one patient because the balloon was advanced too rapidly through the left ventricle. After pericardiocentesis and successful dilatation of the valve the perforation site had to be sutured surgically. Oximetry and opacification of the left atrium showed no left to right shunting after the dilatation in any of the 294 patients.

Vascular surgical intervention on the femoral artery was needed in seven patients (femoral artery intimal flap in three, bleeding at the puncture site in two, a clot in one, and a haematoma in one patient). Surgical intervention was sucessful in all seven patients and there were no chronic circulatory sequelae.

\section{RESULTS AT FOLLOW UP}

At one to three months after operation echocardiography confirmed poor results in 14 patients, whose symptoms remained unchanged. A review of the cineangiograms and video tapes of the procedures showed that in 11 of 14 patients the balloon had not been properly stabilised during inflation and the valve had not been dilated. Six of these patients underwent surgery. Commissural splitting was not found in any of them. In the other five patients balloon dilatation was repeated. These 14 patients were treated in the early part of our experience when simultaneous injection of contrast into the left atrium was not performed.

Two patients died (two and 11 months after operation). One of these patients had developed mitral regurgitation of grade II severity, and the other had no complication related to the operation.

Elective surgery was needed in three out of four patients with grade III mitral regurgitation. Surgery was performed at one year, 18 months, and two years after balloon dilatation. In two of these patients a tear in the anterior mitral leaflet was found and in the third the mitral annulus was dilated (perhaps as a result of overdilatation with $2 \times 20 \mathrm{~mm}$ balloons). One patient was operated on because of a left atrial thrombus at two years, and one because of mitral endocarditis three months after balloon dilatation.

Restenosis (loss of more than $50 \%$ of the increase in valve area), as assessed by repeat catheterisation, was found in seven patients between six months and two years of follow up. Three had surgery and four had repeat balloon 


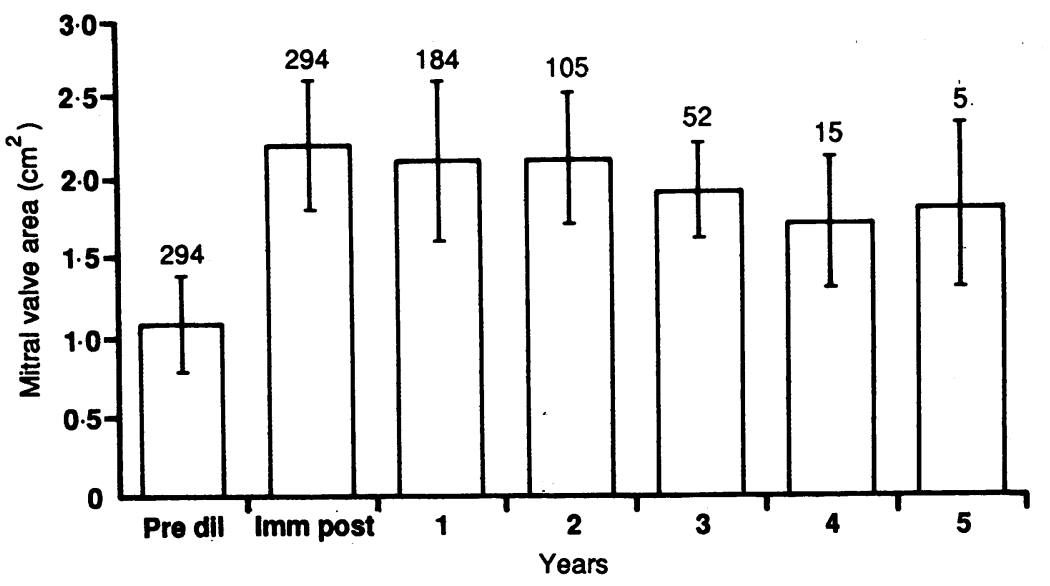

Figure 4 Mitral valve area as determined by cross sectional echocardiography before, (Pre dil) immediately after (Imm post), and one to five years after balloon dilatation of the mitral valve.

dilatation of the valve. In 19 patients atrial fibrillation was converted to sinus rhythm four to 15 (mean 5 (SD 4)) months after dilatation.

The cardiothoracic ratio decreased after the first six months from $0.5(0.09)$ to $0.46(0.1)$ $(p<0.05)$ in 210 patients and did not change further during follow up. Peripheral systemic emboli occurred in four patients during follow up but these had no serious sequelae. Cross sectional echocardiography showed persistence of the achieved mitral valve area during follow up (fig 4). Symptoms improved in most patients during the five year period after balloon dilatation (fig 5). By three years after operation four patients returned to New York Heart Association class IV. Three of them underwent mitral valve replacement and one mitral and aortic valve replacement.

\section{Discussion}

This study reports our experience with transarterial balloon dilatation of the mitral valve in 294 patients, most of whom were young and had a favourable valve anatomy. The procedural mortality of $0 \cdot 7 \%, 2 \cdot 3 \%$ incidence of

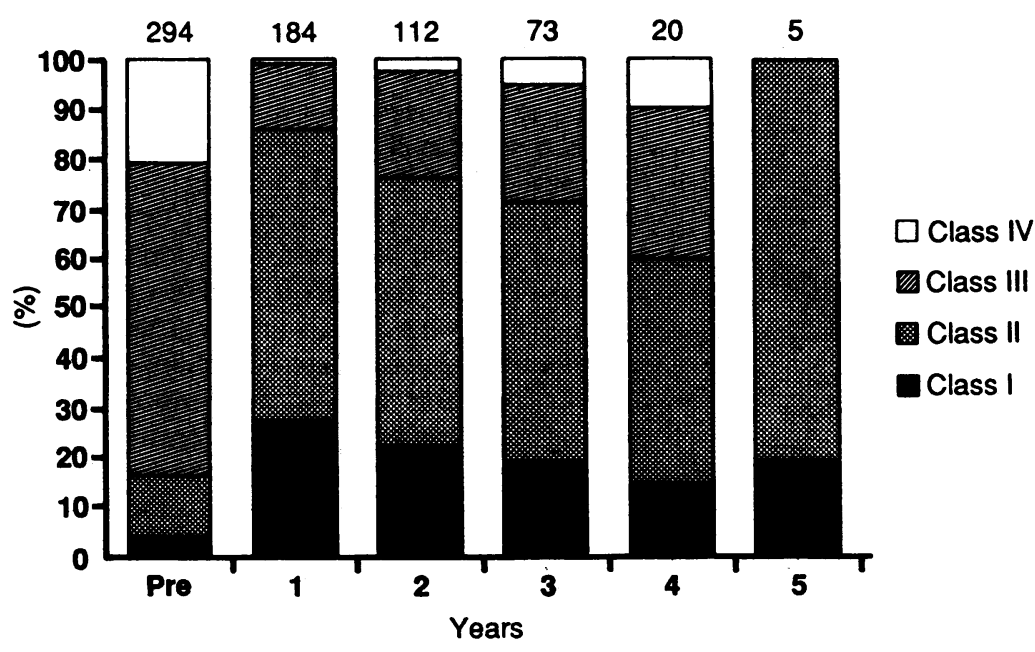

Figure 5 Changes of New York Heart Association functional classes after balloon dilatation of the mitral valve over a five year period. severe mitral regurgitation, and $2 \%$ incidence of embolism were similar figures to those reported after a transvenous approach. ${ }^{12-14} \mathrm{With}$ the advent of transoesophageal echoradiography, which became available in our hospital only in 1991, the risk of embolism should decrease because of better detection of atrial thrombi. There was local injury of the femoral artery in $2 \%$ of our patients. This complication is specific to the transarterial approach and it is regarded as the major disadvantage of this technique.

The atrial septum may be damaged after balloon dilatation of the mitral valve. Although there have been reports that significant left to right shunting leads to an acquired Lutembacher syndrome, ${ }^{1516}$ it is generally considered that these shunts are of little haemodynamic importance. Left to right shunting was found in $62 \%$ of patients immediately after transvenous balloon dilatation with two balloons and in $48 \%$ of patients at six months after the procedure. ${ }^{17}$ After the Inoue mitral balloon procedure left to right shunting, as detected by Doppler colour flow imaging, was found in $15 \%$ of patients immediately after and in $4 \%$ of patients one year after the procedure. ${ }^{18} \mathrm{With}$ the transarterial approach the balloon does not cross the atrial septum and damage to the septum is minimised. We found no evidence of left to right shunting in any of our 294 patients by oximetry. Petrossian et al calculated the mitral valve area after transvenous balloon dilatation of the mitral valve in 13 patients before and after occlusion of the septal hole with a balloon. ${ }^{19}$ The calculated area was larger before than after occlusion. Even when oximetry does not show important shunting the calculated area after transvenous balloon dilatation of the mitral valve may overestimate the real area because of the atrial communication. This finding may mean that some reported data on transvenous balloon dilatation of the mitral valve may not be accurate because the calculation was done without the occlusion of the atrial septal hole.

The transarterial approach aids stabilisation of the ballons at the valve orifice during dilatation. Also simultaneous opacification of the left atrium monitors the events during the balloon inflation and avoids the false impression of valve dilatation that may occur when a resistant orifice causes ejection of the balloons from the orifice.

The major advantages of the transarterial approach are avoidance of injury to the atrial septum and good positioning of the balloon at the orifice. The Inoue balloon dilatation system, however, offers similar advantages and is easier to perform. The Inoue procedure must be compared with the transarterial approach to judge which technique is better.

Most of our patients experienced a symptomatic improvement that was maintained over the follow up period of up to five years. The results of this study support the use of balloon dilatation of the mitral valve for patients with mitral stenosis and pliant valves.

We thank Professor Brian C Morton MD from the University of Ottawa Heart Institute for reviewing this paper and Ms Roxanne Prud'Homme for secretarial assistance. 
1 Inoue $\mathrm{K}$, Owaki T, Nakamura T, Kitamura F, Miymoto N. Clinical application of transvenous mitral commissurotomy by a new balloon catheter. $J$ Thorac Cardiovasc Surg 1984;87:394-402.

2 Lock JE, Khalilullah M, Shrivastava S, Bahl V, Keane JF. Percutaneous catheter commissurotomy in rheumatic mitral stenosis. N Engl J Med 1985;313:1515-8.

3 Zaibag M, Ribeiro PA, Kasab S, Fagih MR. Percutaneous double balloon mitral valvotomy for

4 Palacios A, ed. Atlas of 2-dimensional echocardiography. New York: York Medical Books, 1983:93.

5 Sellers RD, Levy MJ, Amplatz $K$, Lillehei CW. Left retrograde cardiac angiography in acquired cardiac disease. Technic indication and interpretation in 700 cases. Am J Cardiol 1964;14:437-47.

6 Babic UU, Pejcic P, Djuristic Z, Vucinic M, Grujicic S. Percutaneous transarterial balloon valvuloplasty for Percutaneous transarterial balloon valvuloplasty
mitral valve stenosis. Am J Cardiol 1986;57:1101-4.

7 Babic UU, Dorros G. Pejcic P, et al. Percutaneous mitral valvuloplasty: retrograde, transarterial double-balloon technique utilizing the transseptal approach. Cathet Cardiovasc Diagn 1988;14:222-37.

8 LaFarge CG, Miettinen OS. The estimation of oxygen consumption. Cardiovas Res 1970;4:23-30.

9 Carabello BA, Grossman W. Calculation of stenotic valve orifice area. In: Grossman W, ed. Cardiac catheterization and angiography. Philadelphia: Lea and Fibiger, 1986: 143,154

10 Hubbard WN, Hine AL, Rubens M, Donaldson RM. Visualization of left atrial thrombi by coronary arteriography. Cathet Cardiovasc Diagn 1987;13:22-5.
11 Medina A, Bethencourt A, Coello I, et al. Combined percutaneous mitral and aortic valvuloplasty. $\mathrm{Am} J$ Cardiol 1989;64:620-4.

12 Ruiz CE, Alen JW, Lau FYK. Percutaneous double-balloon valvotomy for severe rheumatic mitral valve stenosis. $A m$ J Cardiol 1990;65:473-7.

13 Nobuyoshi M, Hamasaki N, Kimura T, et al. Indications, complications, and short-term clinical outcome of percutaneous transvenous mitral commissurotomy. Circulation 1989;80:782-92.

14 Vahanian A, Michel PL, Cormier B, et al. Results of percutaneous mitral commissurotomy in 200 patients. $\mathrm{Am}$ J Cardiol 1989;63:847-52.

15 Goldberg N, Roman CF, Cha SD. Right to left interatrial shunting following balloon mitral valvuloplasty. Cathet Cardiovasc Diagn 1989;16:133-5.

16 Sadaniantz A, Luttmann C, Shulman RS, Block PC, Sadaniantz A, Luttmann C, Shulman RS, Block PC,
Schachne J, Thompson PD. Acquired Lutembacher Schachne J, Thompson PD. Acquired Lutembacher
syndrome or mitral stenosis and acquired atrial septal syndrome or mitral stenosis and acquired atrial septal
defect after transseptal mitral valvuloplasty. Cathet Cardiovasc Diagn 1990;21:7-9.

17 Cequier A, Bonan R, Serra A, et al. Left-to-right shunting after percutaneous mitral valvuloplasty. Circulation 1990; 81:1190-7.

18 Ishikura F, Nagata S, Yasuda S, Yamashia N, Miyatake K. Residual atrial septal perforation after percutaneous transvenous mitral commissurotomy with Inoue balloon catheter. Am Heart J 1990;120:873-8.

19 Petrossian GA, Ziskin AA, Tuscu ME, Block PC, Palacios I. Atrial septal occlusion improves the accuracy of valve area determination following mitral balloon valvotomy [abstract]. Circulation 1990;82 (suppl III):498. 